\title{
Modelling of short synthetic antifreeze peptides: insights into ice-pinning mechanism
}

$\underline{\text { Enrico Gandini }^{\mathrm{a}}}{ }^{\text {, Maurizio Sironi }}{ }^{\dagger, \mathrm{t}, \mathrm{b}}$ and Stefano Pieraccini ${ }^{\dagger}, \mathrm{a}, \mathrm{b}$

\begin{abstract}
Organisms living in icy environments produce antifreeze proteins to control ice growth and recrystallization. It has been proposed that these molecules pin the surface of ice crystals, thus inducing the formation of a curved surface that arrests crystal growth. Such proteins are very appealing for many potential applications in food industry, material science and cryoconservation of organs and tissues. Unfortunately, their structural complexity has seriously hampered their practical use, while efficient and accessible synthetic analogues are highly desirable. In this paper, we used molecular dynamics based techniques to model the interaction of three short antifreeze synthetic peptides with an ice surface. The employed protocols succeeded in reproducing the ice pinning action of antifreeze peptides and the consequent ice growth arrest, as well as in distinguishing between antifreeze and control peptides, for which no such effect was observed. Principal components analysis of peptides trajectories in different simulation settings permitted to highlight the main structural features associated to antifreeze activity. Modeling results are highly correlated with experimentally measured properties, and insights on ice-peptide interactions and on conformational patterns favoring antifreeze activity will prompt the design of new and improved antifreeze peptides.
\end{abstract}

\section{Keywords}

- $\quad$ Antifreeze peptides

- Antifreeze protein analogues

- Ice-peptide interactions

- Ice-pinning mechanism

- Growing ice simulation

- Principal Component Analysis

a.Dipartimento di Chimica, Università degli Studi di Milano, Via Golgi 19, 20133 Milano, Italy.

b.Istituto di Scienze e Tecnologie Chimiche “G. Natta” (SCITEC-CNR), CNR, and INSTM, UdR Milano, Via Golgi 19, 20133 Milano, Italy.

†Contacts: stefano.pieraccini@unimi.it,maurizio.sironi@unimi.it Supplementary Materials are available. 


\section{Introduction}

Antifreeze Proteins (AFPs) are a class of structurally diverse proteins that protect different species of living organisms from fatally freezing in icy environments [1], [2]. AFPs depress the freezing point of water in a kinetic, non-colligative manner, caused by AFP adsorption to specific surface planes of seed ice crystals [3]. Moreover, AFPs inhibit Ostwald ripening recrystallization of ice, preventing the growth of larger crystals and the concomitant shrinking of smaller ones[4]. The action of AFPs is usually rationalized with the adsorption-inhibition model. It assumes that proteins bind irreversibly to ice thus arresting the growth of the crystal at supercooled conditions through the creation of a metastable curved ice surface, according to the Kelvin effect [5].

Because of their unique capability in controlling ice formation, AFPs are very attractive for potential practical applications including food storage, anti-icing coatings for vehicles and infrastructure and cryopreservation of cells and tissues [6], [7]. Their potential uses has prompted interest in research and led to numerous experimental and computational studies [8]-[15]. However, the molecular complexity, the limited availability and the consequent costs, hamper progress toward AFPs practical application [16]-[18]. Many different AFPs have been identified and categorized according to their structure and their binding specificity. Among them, type I AFPs have been widely studied, particularly the liver-isoform from Winter Flounder (HPLC6). Winter Flounder AFP (wfAFP) is a monomeric, alanine-rich protein, composed of 37 residues, with three 11-residues repeats (TA2NA7).

Even though the antifreeze effect brought about by wfAFP is moderate when compared to that of other AFPs, it is appealing for practical applications because of its simple structure and relatively short sequence.

wfAFP mechanism of action was thoroughly studied both at the experimental and computational level [19]-[25]. It was demonstrated that wfAFP binds preferentially along the $\langle 01 \overline{1} 2\rangle$ axis of the pyramidal ice plane [26], [27]. The hydrophobic face of wfAFP is presented to the pyramidal ice plane, and the interaction mechanism is similar to the hydrophobic solvation effect [23].

Efficient synthetic analogues mimicking the effects of AFPs are highly desirable. Three 12-residues analogues of wfAFP (see Table 1) were synthesized and successfully applied for the fabrication of anti-icing surfaces by Zhang et al.[7].

The present paper is focused on the application of Molecular Dynamics based techniques to investigate at the atomic level the antifreeze activity of the three aforementioned peptidic wfAFP analogues and to model their binding to ice surface and their mechanism of action.

\section{Methods}

\section{Modelling of the Peptides}

Table 1: Amino acid sequences of three antifreeze peptides considered in the present study. Residues that differ from original wfAFP are highlighted. The table also includes molecular weights, and reported average freezing temperatures of water droplets on peptide-coated silicon wafers.

\begin{tabular}{|c|c|c|c|}
\hline Peptide & Sequence & $\begin{array}{c}\text { Molecular } \\
\text { Weight } / \mathrm{Da}\end{array}$ & $\begin{array}{c}\text { Average Freezing } \\
\text { Temperature } /{ }^{\circ} \mathrm{C}\end{array}$ \\
\hline $1-1$ & DTASDAAAAAAL & 1047 & -11.9 \\
\hline $1-2$ & DTASDAKAAAEL & 1162 & -16.3 \\
\hline $1-3$ & DTASDAFAAAAL & 1123 & -10.8 \\
\hline
\end{tabular}

Three 12 residues peptides derived from wfAFP have been considered (amino acid sequences are reported in Table 1). Peptide $1-1$ is composed of the first 12 residues of wfAFP. The wfAFP crystal structure was obtained from PDB id 
1WFA [20]. The relevant residues were kept, all the others were manually removed. Peptides 1-2 and 1-3 present residue mutations that were designed to improve their antifreeze properties [7]. In particular, for Peptide 1-2, Alanine 7 was mutated to a Lysine, and Alanine 11 to a Glutamic Acid, in order to form an intra-molecular saline bridge to increase helicity. For Peptide 1-3, Alanine 7 was mutated to a Phenylalanine, in order to improve hydrophobic interactions with ice. The structural models of Peptides 1-2 and 1-3 were obtained through residue mutation with UCSF Chimera Rotamer tool [28], [29]. The peptides were then protonated with Gromacs 5.0.4 [30], and described with CHARMM 27 Force Field [31], which shows the best performance in modeling ice-protein interactions along with TIP4P/Ice water model [32], [33]. The three antifreeze peptides, and control peptides dodeca-Glycine (G12) and dodeca-Alanine (A12), were then subjected to three kinds of simulations.

\section{Simulations in a water box}

The peptides were inserted into a cubic box of side $4.5 \mathrm{~nm}$. The box was solvated with TIP4P/Ice [32] water molecules, and brought to charge neutrality with $1 \mathrm{M}$ concentration of $\mathrm{Na}$ and $\mathrm{Cl}$ ions. The system was then subjected to restrained relaxation and NVT and NPT equilibration, at temperature $275 \mathrm{~K}$ and 1 bar pressure. After the equilibrations, the structure restraints were removed, and the peptides were subjected to $1.5 \mu$ molecular dynamics simulations at NPT conditions.
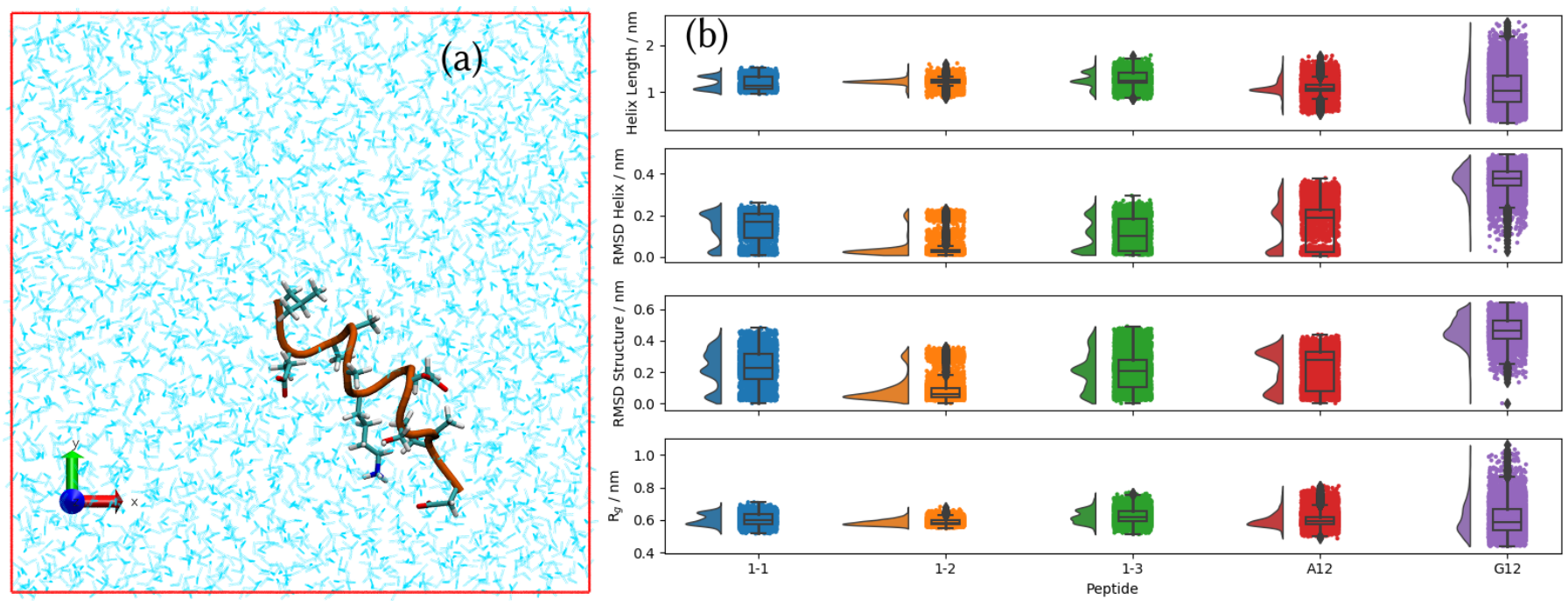

Figure 1: (a): Final frame of Peptide 1-2 water box simulation. Liquid waters are in semi-transparent line representation. Protein backbone is in orange tube representation, and side chains are in licorice representation colored by atom type. (b): Distributions of four structural properties calculated throughout the water box simulations: radius of gyration, structural RMSD, RMSD from an ideal $\alpha$ helix, helix length.

\section{Simulations on a fixed ice surface}

A large unit cell of $\mathrm{l}$ proton-disordered Ice was generated with Genlce tool [34]. A $2.5 \mathrm{~nm}$ thick slab of ice, exposing the pyramidal plane, was obtained with Vesta [35]. Peptide 1-1 was manually placed on the pyramidal plane along the $<01 \overline{1} 2>$ axis, with the hydrophobic residues facing ice [23]. Peptides 1-2 and 1-3 were generated in loco through mutation with Chimera. The ice-peptide systems were then inserted in $5.7 \times 5 \times 16 \mathrm{~nm}$ box. Water molecules and ions were then added, but the last $5 \mathrm{~nm}$ of the box in the $\mathrm{Z}$ direction were left empty, in order to create an ice - peptide water - vacuum system as described by Mochizuki et al. [36] and shown in Figure 3(b).

Restraints were added to the protein $\mathrm{C}_{\alpha}$ atoms and to the ice oxygens, in order to carry out system relaxations and NVT equilibrations at $275 \mathrm{~K}$. Afterwards, the protein restraints were removed, and the systems were subjected to 100 ns molecular dynamics simulations.

\section{Simulations on a growing ice surface}

The ice - peptide - water - vacuum systems were prepared in the same way as described above for the fixed ice simulations. In this new set of simulations, the NVT equilibration and the production molecular dynamics simulation 
were carried out at $248 \mathrm{~K}$, below the reported $270 \mathrm{~K}$ freezing point of TIP4P/Ice water model freezing point [32], so that ice growth could be observed within the 850 ns long production trajectories. A simulation without any peptide was included for control.

\section{Trajectory Analysis}

All structural renderings were performed with VMD [37]. Secondary structures were calculated using DSSP [38] algorithm as implemented in MDTraj [39]. Structural RMSD, radius of gyration, helix length and helicity were calculated with Gromacs rmsd, gyrate, and helix tools [30]. Root Mean Square Fluctuation (RMSF) calculations were performed with MDAnalysis [40], [41]. Protein-Ice Contact Surface (PICS) were calculated through Solvent Accessible Surface Area (SASA) with MDAnalysis. All graphs were created with Python plotting libraries [42]-[44].

Principal Component Analysis (PCA) [45], [46] was applied to antifreeze and control peptides trajectories in the three sets of simulations. In order to apply PCA, for each simulation set, we obtained the coordinates of $\mathrm{C}_{\alpha}$ atoms throughout each peptide trajectory, using Gromacs trjconv tool, and we aligned $\mathrm{C}_{\alpha}$ geometries with MDAnalysis. We then used ENCORE [47] to perform PCA on $C_{\alpha}$ trajectories of all peptides, for each simulation set. ENCORE concatenates $C_{\alpha}$ trajectories of all peptides (possible since every peptide has the same number of $C_{\alpha}$ atoms, twelve in this case), and then applies PCA algorithm implemented in scikit-learn [48] to the concatenated $\mathrm{C}_{\alpha}$ coordinates. Since the $C_{\alpha}$ atoms trajectories are concatenated in a single coordinate matrix, PCA algorithm calculates the Principal Components (PCs) that describe the total conformational variance across all the peptides. When PCA is applied on concatenated trajectories of different peptides, the calculated PCs highlight conformational differences and similarities between the peptides. Each PC describes a certain percentage of the total conformational variance across all trajectories. The higher this percentage variance, the most important is the global motion described by the PC.

\section{Results and Discussion}

The water box set of simulations was tested for conformational stability and helicity of the peptides, which in case of wfAFP is highly correlated with antifreeze activity [1].

To test whether the peptides present the same characteristic helical structure of the original wfAFP, secondary structures were calculated with the DSSP algorithm [38] throughout the trajectories (Supplementary Figures 3-5). All the peptides present high degrees of helicity. Peptide 1-2 is more helical than the other peptides, in good agreement with reported circular dichroism spectra [7]. Moreover, four structural properties of the conformational ensembles of the antifreeze and control peptides (Radius of gyration, Structural RMSD, RMSD from ideal helix, helix length) were calculated throughout the trajectories and are reported in Figure 1(b). The four chosen properties are measures of conformational stability and resemblance of the peptides with an ideal $\alpha$ helix. In particular, in agreement with experimental results, Peptide 1-2 shows greater propensity for helical structure than Peptides 1-1 and 1-3. 

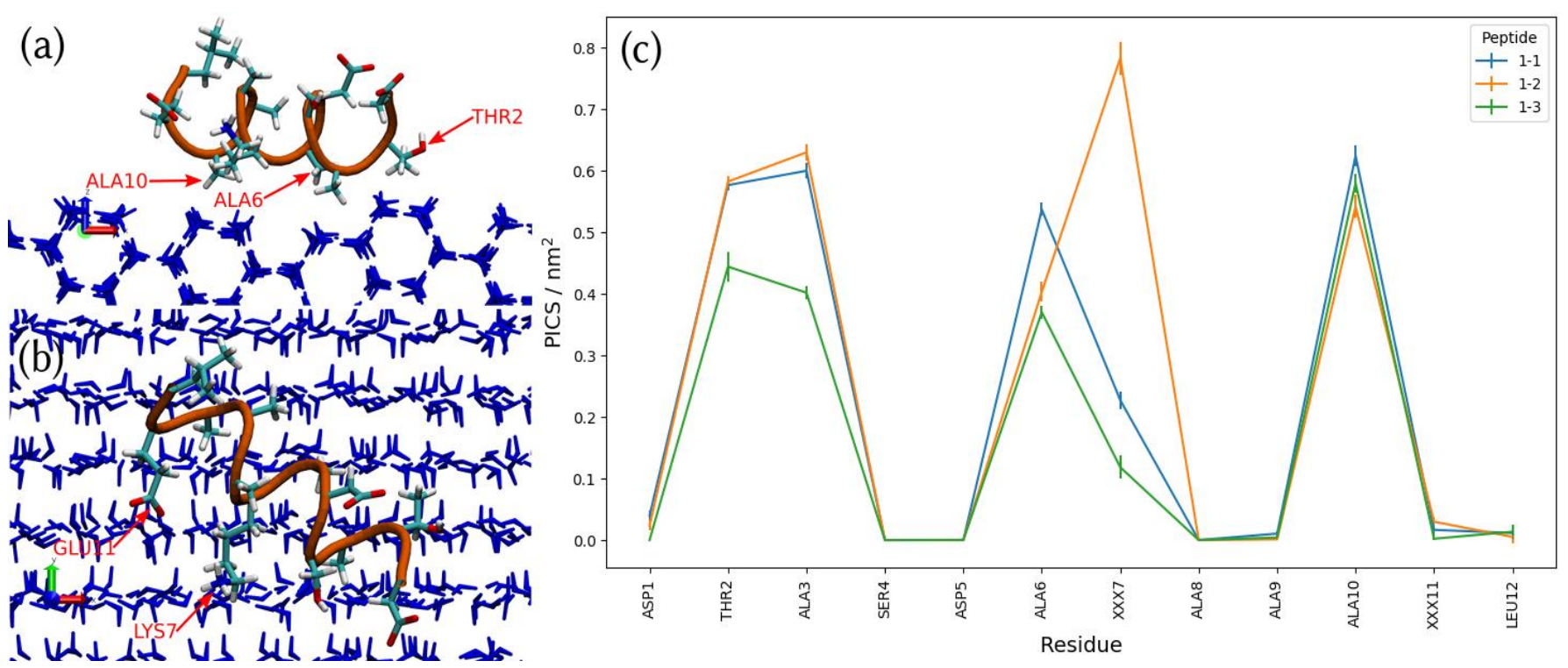

Figure 2: $(a, b)$ : two views of Peptide 1-2 fixed ice simulation final frame. Restrained ice is in blue licorice representation. The backbone is in orange tube representation, and side chains are licorice colored by atom type. (c): Average PICS of antifreeze peptides with bootstrapped error bars, calculated during fixed ice simulations. Residues which are different among the peptides are labelled XXX.

$\mathrm{C}_{\alpha}$ atoms RMSF were also calculated on water box trajectories (Supplementary Figure 6). Confidence intervals are $2 \times$ Standard Error of the Mean (SEM) calculated with bootstrapping [49], [50]. The residues of the three antifreeze peptides have low RMSF values, indicating limited flexibility. Peptide 1-2 has the lowest RMSFs. RMSF values were also calculated for the fixed ice simulations (Supplementary Figure 10): they are even lower than in water box simulations, indicating that the presence of the ice surface has a stabilizing effect on peptides conformations. RMSF values does not significantly differ between antifreeze peptides in the fixed ice simulations. The antifreeze mechanism and the nature of the ice-wfAFP interactions are the subject of many hypotheses [21]. It is believed that both direct interactions between wfAFP with ice surface, and interactions mediated by water molecules at ice-protein interface play a role in the antifreeze activity. Average PICS was calculated and results are presented in Figure 2(c). Residues with high PICS values are the ones that interact more steadily with ice. THR2, ALA6 and ALA10 interactions are relevant in all three peptides, in good agreement with the observations of Kun and Mastai [51]. Residue 7, which is an ALA for Peptide 1-1, a LYS for Peptide 1-2, and a PHE for Peptide 1-3, also exhibits a large PICS for all the three peptides, but it is significantly larger for Peptide 1-2, suggesting that LYS side chain favors the interaction. Close inspection of the trajectory reveals that the interaction is brought about mostly by carbon atoms of the LYS side chain, and not by its positively charged group, which interacts either with GLU11 or the solvent molecules, as shown in Figure 2(a), which presents two different views of the final trajectory frame of Peptide 1-2 on fixed ice.

The three antifreeze peptides remained firmly attached to the ice surfaces for the duration of the fixed ice simulations, whereas non-antifreeze Peptide G12, after few nanoseconds, starts to change its secondary structure and to detach from the ice surface. Non-antifreeze Peptide A12 keeps its helical structure throughout the fixed ice simulation. Structural properties of the five peptides calculated throughout the fixed ice trajectories are reported in Supplementary Figure 9.

In order to assess the ability of antifreeze peptides to inhibit ice growth, a set of simulations below TIP4P/Ice freezing point has been performed.

Figure 3 presents different views of the final trajectory frame of Peptide 1-2 growing ice simulation. The peptide is firmly attached to the original restrained ice surface, and it induces the formation of a curved ice front from the liquid water molecules in the course of the simulation, as observed in previous computational studies [12], [52]. No ice growth above the peptide is detected. Quantification of ice formed during growing ice simulations was performed with CHILL+ algorithm [53], and results are shown in Figure 3(c). Ice starts growing immediately in control simulations 
performed without peptide: all water is turned into ice after $150 \mathrm{~ns}$. In simulation performed with non-antifreeze peptide G12, ice starts growing more slowly, and the growth is complete after 500 ns. Peptide G12 is completely enclosed in a block of ice formed by initially liquid water. In presence of non-antifreeze Peptide A12, ice growth is slower, and it is complete after $800 \mathrm{~ns}$. Ice is not able to grow above the antifreeze peptides. The total quantity of ice formed with antifreeze peptides is around $10 \%$, throughout the whole 850 ns simulations. Visual inspection of the growing ice trajectories along with the application of CHILL+ algorithm confirmed that the synthetic analogues of wfAFP can shape ice surface inducing the formation of a curved ice front and consequently block ice growth with a mechanism compatible with the Kelvin effect.

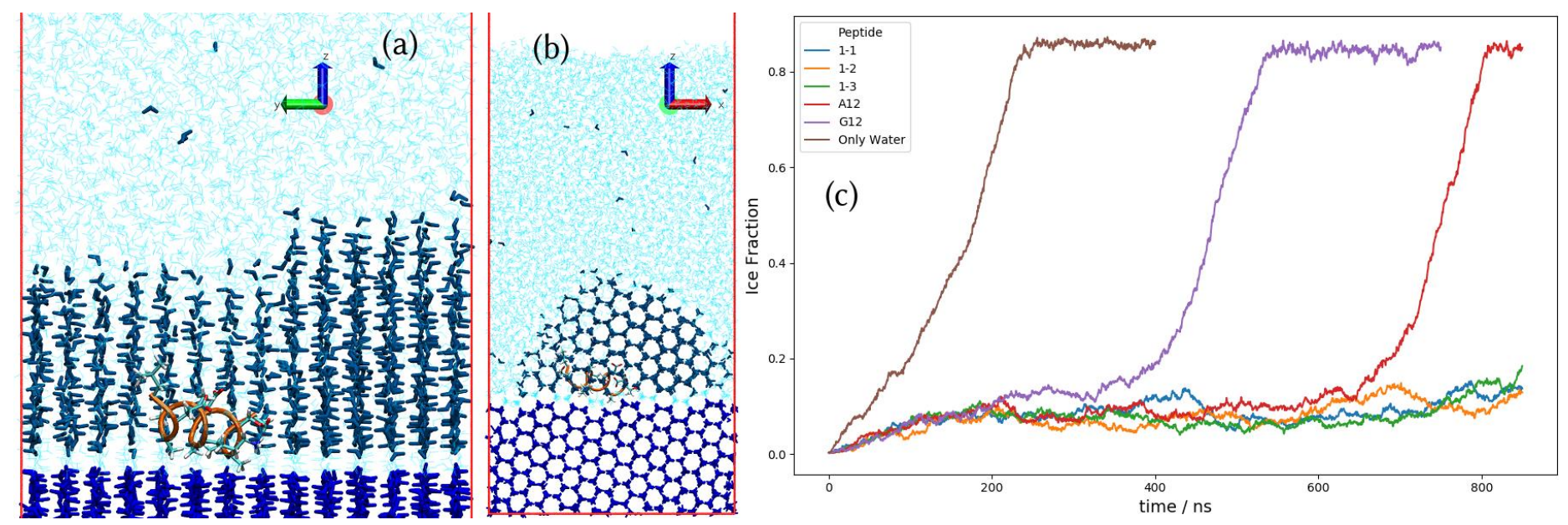

Figure 3: $(a, b)$ : two views of Peptide 1-2 growing ice simulation final frame. Restrained ice is in dark blue licorice representation, whereas ice that was formed from unrestrained water during the simulation is in lighter blue licorice. Liquid water molecules are represented as semi-transparent lines. Protein backbone is in orange tube representation, and side chains are in licorice colored by atom types. (c): Fraction of ice formed from unrestrained water throughout the growing ice simulations.

\section{Principal Component Analysis of Conformational Ensembles}

Principal Component Analysis (PCA) is a general purpose statistical procedure [45], [46] that has often been applied successfully to study molecular dynamics trajectories [25], [50], [54], [55].

When studying a series of similar peptides or proteins, it is often interesting to take into account the conformational effects that are induced by residue mutations which can either conserve or modify the conformations of the protein under consideration. In particular, for antifreeze proteins mutation analysis is very important, since interactions with ice strongly depend on protein conformations [56], [57]. PCA is a useful technique to compare a series of same-length similar peptides or proteins. In a single graph, it shows differences in the global motions that are caused by residue mutations, and highlights structural peculiarities of the most effective peptides.

In the present work, conformational ensembles of antifreeze peptides, and of non-antifreeze peptides G12 and A12, were compared through PCA using the algorithm as implemented in ENCORE [47]. The software concatenates $\mathrm{C}_{\alpha}$ atoms coordinates of all peptides throughout the trajectories in a single coordinate matrix, and performs PCA.

Figure 4(a) shows $\mathrm{C}_{\alpha}$ atoms coordinates throughout the water box simulations projected onto the first two PCs, that together account for $47 \%$ of the total motions. Antifreeze peptides, and non-antifreeze Peptide A12, cover a similar area, thus confirming that they explore a similar conformational ensemble. As expected, Peptide G12 covers a much larger area, since it does not have a preferred secondary structure.

PCA was then applied to $\mathrm{C}_{\alpha}$ coordinates throughout the fixed ice simulations, and results are shown in Figure 4(b). The first two PCs account for $66 \%$ of the total motions, which is more than the variance explained by the first two PCs in the water box simulations, because of the stabilizing presence of the ice surface. Antifreeze peptides, and control peptide A12, occupy an even smaller area compared to that covered by G12, pointing out that most of the total variance explained by the two main PCs comes from G12, whereas the antifreeze peptides are much stabilized by the presence of the ice surface. Even though A12 is not an antifreeze peptide, Alanine residues are known to interact with ice [23], [58]. So, A12 is stabilized by the ice surface, and its area is the same size as that of antifreeze peptides. 
Results of the application of PCA to growing ice simulations are shown in Figure 4(c). The variance explained by PC1 is $67 \%$, whereas that explained by PC2 is $17 \%$. The total motions explained by the first two PCs is $83 \%$, even higher than the fixed ice simulation, since now the ice growth further limits the conformational freedom of peptide G12. PC1 is responsible for the separation of G12 from antifreeze peptides, whereas PC2 is able to differentiate between Peptide 1-2 and Peptides 1-1 and 1-3. Conformations representative of the extreme PC values were extracted from the trajectories and reported onto the PC graph. PC1 separates a helical structure from a disordered structure. Interestingly, the much smaller global motion represented by PC2 separates a perfect helical structure from a helical structure with the C-term residues outstretched. A close inspection of Peptide 1-2 structure with outstretched C-term residues, suggests that the elongation may be brought about by GLU11 interaction with liquid water. LEU12, though outstretched, is still interacting with the ice surface, as well as the aliphatic carbons of LYS7, whereas the positively charged group of LYS7 side chain interacts with liquid water. Non-antifreeze Peptide A12 has PC1 values similar to those of antifreeze peptides, thus confirming that antifreeze peptides and A12 explore a similar conformational ensemble, and have similar secondary structures. On the other hand, Peptide A12 has PC2 values similar to those of Peptide 1-2.
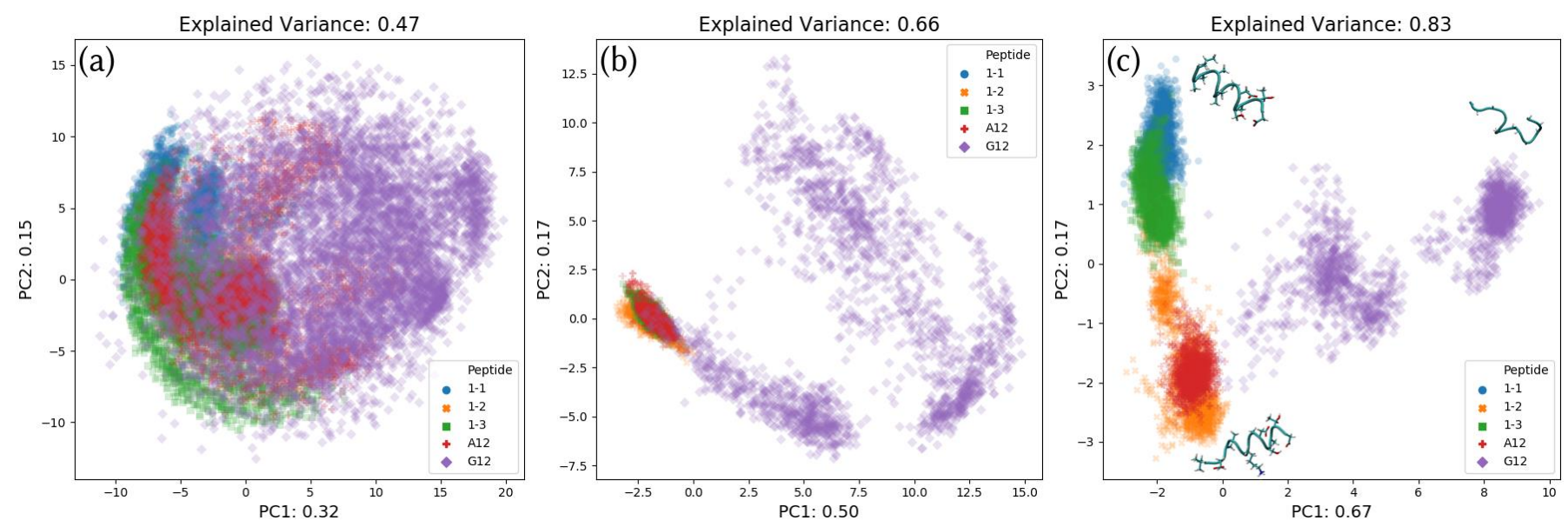

Figure 4: C $\alpha$ coordinates projected onto the first two PCs of water box simulations (a), fixed ice simulations (b), and growing ice simulations (c). Structures which are representative of the PCs extremes are also inserted on (c).

\section{Conclusions}

Three synthetic analogues of wfAFP, which have shown experimentally measureable ice growth inhibition activity, were the subject of computational modelling and simulation. Three simulation setups were devised, in order to analyse different molecular properties that affect antifreeze activity. Simulations in a water box were able to reproduce the experimentally observed conformational and secondary structure stability of the three antifreeze peptides. Simulations on a fixed ice surface pointed out the presence of stabilizing interactions between the antifreeze peptides and an ice slab exposing the pyramidal plane. Simulations on a growing ice surface were able to reveal an icegrowth blocking effect for the three antifreeze peptides. PCA of $C_{\alpha}$ atoms coordinates pointed out differences in the global motions of antifreeze peptides from non-antifreeze Peptide G12.

Secondary structure of Peptide A12 is similar to that of antifreeze peptides, and PCA confirmed that global motions of A12 are similar to those of antifreeze peptides. Anyway, helical propensity is not, in itself, a guarantee of antifreeze activity [56]. CHILL+ measurements of ice formed during simulations confirm that A12 is unable to block ice growth, even though it is structurally similar to antifreeze peptides.

When applied to the growing ice simulation, PCA was also able to extract a structural pattern peculiar to the conformational ensemble of antifreeze Peptide 1-2 that will be useful to design new synthetic analogues of wfAFP. The protocol that was described in this work is useful to analyze the conformational properties and antifreeze activity of series of short peptides. Even though peptides derived from wfAFP may not present the greatest antifreeze activity 
at low concentrations, their short chains and simple structures make them promising for large-scale synthesis and practical applications.

\section{Acknowledgements}

We acknowledge the CINECA award under the ISCRA initiative, for the availability of high performance computing resources and support (application code: HP10C8VXWW).

We thank Prof. Sara Pellegrino, Prof. Matteo Pecchiari, Prof. Fabio Acocella, and Dr. Giuliano Zanchetta, for helpful discussion.

\section{References}

[1] M. Bar Dolev, I. Braslavsky, e P. L. Davies, "lce-Binding Proteins and Their Function», Annual Review of Biochemistry, vol. 85, n. 1, pagg. 515-542, giu. 2016, doi: 10.1146/annurev-biochem-060815-014546.

[2] Bowles D. J. et al., "Structure and function of antifreeze proteins", Philosophical Transactions of the Royal Society of London. Series B: Biological Sciences, vol. 357, n. 1423, pagg. 927-935, lug. 2002, doi: 10.1098/rstb.2002.1081.

[3] L. L. C. Olijve et al., «Blocking rapid ice crystal growth through nonbasal plane adsorption of antifreeze proteins», Proceedings of the National Academy of Sciences, vol. 113, n. 14, pagg. 3740-3745, apr. 2016, doi: 10.1073/pnas.1524109113.

[4] C. A. Knight, D. Wen, e R. A. Laursen, «Nonequilibrium Antifreeze Peptides and the Recrystallization of Ice», Cryobiology, vol. 32, n. 1, pagg. 23-34, feb. 1995, doi: 10.1006/cryo.1995.1002.

[5] P. Wilson, «Explaining thermal hysteresis by the Kelvin effect», CryoLetters, vol. 14, pagg. 31-36, 1993.

[6] I. K. Voets, «From ice-binding proteins to bio-inspired antifreeze materials», Soft Matter, vol. 13, n. 28, pagg. 4808-4823, 2017, doi: 10.1039/C6SM02867E.

[7] Y. Zhang, K. Liu, K. Li, V. Gutowski, Y. Yin, e J. Wang, "Fabrication of Anti-Icing Surfaces by Short $\alpha$-Helical Peptides», ACS Applied Materials \& Interfaces, vol. 10, n. 2, pagg. 1957-1962, gen. 2018, doi: 10.1021/acsami.7b13130.

[8] H. Nada e Y. Furukawa, «Antifreeze proteins: computer simulation studies on the mechanism of ice growth inhibition», Polymer Journal, vol. 44, n. 7, pagg. 690-698, lug. 2012, doi: 10.1038/pj.2012.13.

[9] R. K. Kar e A. Bhunia, «Biophysical and biochemical aspects of antifreeze proteins: Using computational tools to extract atomistic information", Progress in Biophysics and Molecular Biology, vol. 119, n. 2, pagg. 194-204, nov. 2015, doi: 10.1016/j.pbiomolbio.2015.09.001.

[10] A. Hudait, D. R. Moberg, Y. Qiu, N. Odendahl, F. Paesani, e V. Molinero, «Preordering of water is not needed for ice recognition by hyperactive antifreeze proteins», Proceedings of the National Academy of Sciences, vol. 115, n. 33, pagg. 8266-8271, ago. 2018, doi: 10.1073/pnas.1806996115.

[11] A. Hudait, Y. Qiu, N. Oden, e V. Molinero, «Hydrogen-Bonding and Hydrophobic Groups Contribute Equally to the Binding of Hyperactive Antifreeze and Ice-Nucleating Proteins to Ice | Journal of the American Chemical Society", J. Am. Chem. Soc., vol. 141, n. 19, pagg. 7887-7898, 2019, doi: 10.1021/jacs.9b02248.

[12] M. J. Kuiper, C. J. Morton, S. E. Abraham, e A. Gray-Weale, «The biological function of an insect antifreeze protein simulated by molecular dynamics», eLife, vol. 4, mag. 2015, doi: 10.7554/eLife.05142.

[13] S. S. Mallajosyula, K. Vanommeslaeghe, e A. D. MacKerell, «Perturbation of Long-Range Water Dynamics as the Mechanism for the Antifreeze Activity of Antifreeze Glycoprotein», J. Phys. Chem. B, vol. 118, n. 40, pagg. 11696-11706, ott. 2014, doi: 10.1021/jp508128d.

[14] K. Meister et al., "Observation of ice-like water layers at an aqueous protein surface», PNAS, vol. 111, n. 50, pagg. 17732-17736, dic. 2014, doi: 10.1073/pnas.1414188111. 
[15] S. Ebbinghaus, K. Meister, B. Born, A. L. DeVries, M. Gruebele, e M. Havenith, "Antifreeze Glycoprotein Activity Correlates with Long-Range Protein-Water Dynamics», J. Am. Chem. Soc., vol. 132, n. 35, pagg. 12210-12211, set. 2010, doi: 10.1021/ja1051632.

[16] S. Venketesh e C. Dayananda, "Properties, Potentials, and Prospects of Antifreeze Proteins», Critical Reviews in Biotechnology, vol. 28, n. 1, pagg. 57-82, gen. 2008, doi: 10.1080/07388550801891152.

[17] R. E. Feeney e Y. Yeh, "Antifreeze proteins: Current status and possible food uses», Trends in Food Science \& Technology, vol. 9, n. 3, pagg. 102-106, mar. 1998, doi: 10.1016/S0924-2244(98)00025-9.

[18] N. S. Ustun e S. Turhan, «Antifreeze Proteins: Characteristics, Function, Mechanism of Action, Sources and Application to Foods», Journal of Food Processing and Preservation, vol. 39, n. 6, pagg. 3189-3197, 2015, doi: 10.1111/jfpp.12476.

[19] P. Dalal e F. D. Sönnichsen, "Source of the Ice-Binding Specificity of Antifreeze Protein Type I», J. Chem. Inf. Comput. Sci., vol. 40, n. 5, pagg. 1276-1284, set. 2000, doi: 10.1021/ci000449b.

[20] F. Sicheri e D. S. C. Yang, "lce-binding structure and mechanism of an antifreeze protein from winter flounder», Nature, vol. 375, pagg. 427-431, 1995.

[21] M. M. Harding, L. G. Ward, e A. D. J. Haymet, "Type I 'antifreeze' proteins», European Journal of Biochemistry, vol. 264, n. 3, pagg. 653-665, 1999, doi: 10.1046/j.1432-1327.1999.00617.x.

[22] S. Chakraborty e B. Jana, «Conformational and hydration properties modulate ice recognition by type I antifreeze protein and its mutants», Phys. Chem. Chem. Phys., vol. 19, n. 18, pagg. 11678-11689, mag. 2017, doi: 10.1039/C7CP00221A.

[23] A. Wierzbicki, P. Dalal, T. E. Cheatham, J. E. Knickelbein, A. D. J. Haymet, e J. D. Madura, «Antifreeze Proteins at the Ice/Water Interface: Three Calculated Discriminating Properties for Orientation of Type I Proteins», Biophysical Journal, vol. 93, n. 5, pagg. 1442-1451, set. 2007, doi: 10.1529/biophysj.107.105189.

[24] T. Nobekawa e Y. Hagiwara, "Interaction among the twelve-residue segment of antifreeze protein type I, or its mutants, water and a hexagonal ice crystal», Molecular Simulation, vol. 34, n. 6, pagg. 591-610, mag. 2008, doi: 10.1080/08927020801986556.

[25] R. K. Kar e A. Bhunia, «Will It Be Beneficial To Simulate the Antifreeze Proteins at Ice Freezing Condition or at Lower Temperature?», J. Phys. Chem. B, vol. 119, n. 35, pagg. 11485-11495, set. 2015, doi: 10.1021/acs.jpcb.5b04919.

[26] D. Wen e R. A. Laursen, "A model for binding of an antifreeze polypeptide to ice», Biophysical Journal, vol. 63, n. 6, pagg. 1659-1662, dic. 1992, doi: 10.1016/S0006-3495(92)81750-2.

[27] C. A. Knight, C. C. Cheng, e A. L. DeVries, «Adsorption of alpha-helical antifreeze peptides on specific ice crystal surface planes», Biophysical Journal, vol. 59, n. 2, pagg. 409-418, feb. 1991, doi: 10.1016/S0006-3495(91)822342.

[28] E. F. Pettersen et al., "UCSF Chimera-A visualization system for exploratory research and analysis», Journal of Computational Chemistry, vol. 25, n. 13, pagg. 1605-1612, 2004, doi: 10.1002/jcc.20084.

[29] M. V. Shapovalov e R. L. Dunbrack, «A Smoothed Backbone-Dependent Rotamer Library for Proteins Derived from Adaptive Kernel Density Estimates and Regressions», Structure, vol. 19, n. 6, pagg. 844-858, giu. 2011, doi: 10.1016/j.str.2011.03.019.

[30] D. Van Der Spoel, E. Lindahl, B. Hess, G. Groenhof, A. E. Mark, e H. J. Berendsen, «GROMACS: fast, flexible, and free», Journal of computational chemistry, vol. 26, n. 16, pagg. 1701-1718, 2005.

[31] N. Foloppe e A. D. M. Jr, «All-atom empirical force field for nucleic acids: I. Parameter optimization based on small molecule and condensed phase macromolecular target data», Journal of Computational Chemistry, vol. 21, n. 2, pagg. 86-104, 2000, doi: 10.1002/(SICI)1096-987X(20000130)21:2<86::AID-JCC2>3.0.CO;2-G.

[32] J. L. F. Abascal, E. Sanz, R. García Fernández, e C. Vega, "A potential model for the study of ices and amorphous water: TIP4P/Ice», J. Chem. Phys., vol. 122, n. 23, pag. 234511, giu. 2005, doi: 10.1063/1.1931662.

[33] H. Lee, "Structures, dynamics, and hydrogen-bond interactions of antifreeze proteins in TIP4P/Ice water and their dependence on force fields», PLOS ONE, vol. 13, n. 6, pag. e0198887, giu. 2018, doi: 10.1371/journal.pone.0198887. 
[34] M. Matsumoto, T. Yagasaki, e H. Tanaka, "Genlce: Hydrogen-Disordered Ice Generator», Journal of Computational Chemistry, vol. 39, n. 1, pagg. 61-64, 2018, doi: 10.1002/jcc.25077.

[35] K. Momma e F. Izumi, "VESTA 3 for three-dimensional visualization of crystal, volumetric and morphology data», J App/ Cryst, vol. 44, n. 6, pagg. 1272-1276, dic. 2011, doi: 10.1107/S0021889811038970.

[36] K. Mochizuki e V. Molinero, "Antifreeze Glycoproteins Bind Reversibly to Ice via Hydrophobic Groups», Journal of the American Chemical Society, vol. 140, n. 14, pagg. 4803-4811, apr. 2018, doi: 10.1021/jacs.7b13630.

[37] W. Humphrey, A. Dalke, e K. Schulten, "VMD: Visual molecular dynamics», Journal of Molecular Graphics, vol. 14, n. 1, pagg. 33-38, feb. 1996, doi: 10.1016/0263-7855(96)00018-5.

[38] W. Kabsch e C. Sander, «Dictionary of protein secondary structure: Pattern recognition of hydrogen-bonded and geometrical features», Biopolymers, vol. 22, n. 12, pagg. 2577-2637, dic. 1983, doi: 10.1002/bip.360221211.

[39] R. T. McGibbon et al., "MDTraj: A Modern Open Library for the Analysis of Molecular Dynamics Trajectories», Biophysical Journal, vol. 109, n. 8, pagg. 1528-1532, ott. 2015, doi: 10.1016/j.bpj.2015.08.015.

[40] R. J. Gowers et al., "MDAnalysis: A Python Package for the Rapid Analysis of Molecular Dynamics Simulations», in Proceedings of the 15th Python in Science Conference, 2016, pag. 8.

[41] N. Michaud-Agrawal, E. J. Denning, T. B. Woolf, e O. Beckstein, «MDAnalysis: A toolkit for the analysis of molecular dynamics simulations», Journal of Computational Chemistry, vol. 32, n. 10, pagg. 2319-2327, 2011, doi: $10.1002 / j c c .21787$.

[42] J. D. Hunter, «Matplotlib: A 2D Graphics Environment», Computing in Science Engineering, vol. 9, n. 3, pagg. 9095, mag. 2007, doi: 10.1109/MCSE.2007.55.

[43] «seaborn: statistical data visualization - seaborn 0.10 .0 documentation». https://seaborn.pydata.org/ (consultato feb. 12, 2020).

[44] M. Allen, D. Poggiali, K. Whitaker, T. R. Marshall, e R. A. Kievit, «Raincloud plots: a multi-platform tool for robust data visualization», Wellcome Open Res, vol. 4, pag. 63, apr. 2019, doi: 10.12688/wellcomeopenres.15191.1.

[45] S. Wold, K. Esbensen, e P. Geladi, «Principal Component Analysis», pag. 16.

[46] H. Abdi e L. J. Williams, «Principal component analysis», WIREs Comp Stat, vol. 2, n. 4, pagg. 433-459, lug. 2010, doi: 10.1002/wics.101.

[47] M. Tiberti, E. Papaleo, T. Bengtsen, W. Boomsma, e K. Lindorff-Larsen, «ENCORE: Software for Quantitative Ensemble Comparison», PLOS Computational Biology, vol. 11, n. 10, pag. e1004415, ott. 2015, doi: 10.1371/journal.pcbi.1004415.

[48] F. Pedregosa et al., "Scikit-learn: Machine Learning in Python», Journal of Machine Learning Research, vol. 12, n. 85, pagg. 2825-2830, 2011.

[49] A. Mitra e D. Sept, «Taxol Allosterically Alters the Dynamics of the Tubulin Dimer and Increases the Flexibility of Microtubules», Biophysical Journal, vol. 95, n. 7, pagg. 3252-3258, ott. 2008, doi: 10.1529/biophysj.108.133884.

[50] F. Dapiaggi, S. Pieraccini, e M. Sironi, «In silico study of VP35 inhibitors: from computational alanine scanning to essential dynamics», Molecular BioSystems, vol. 11, n. 8, pagg. 2152-2157, 2015, doi: 10.1039/C5MB00348B.

[51] H. Kun e Y. Mastai, «Activity of short segments of Type I antifreeze protein», Peptide Science, vol. 88, n. 6, pagg. 807-814, 2007, doi: 10.1002/bip.20844.

[52] P. M. Naullage, Y. Qiu, e V. Molinero, «What Controls the Limit of Supercooling and Superheating of Pinned Ice Surfaces?», The Journal of Physical Chemistry Letters, vol. 9, n. 7, pagg. 1712-1720, apr. 2018, doi: 10.1021/acs.jpclett.8b00300.

[53] A. H. Nguyen e V. Molinero, «Identification of Clathrate Hydrates, Hexagonal Ice, Cubic Ice, and Liquid Water in Simulations: the CHILL+ Algorithm», J. Phys. Chem. B, vol. 119, n. 29, pagg. 9369-9376, lug. 2015, doi: 10.1021/jp510289t.

[54] G. G. Maisuradze, A. Liwo, e H. A. Scheraga, "Principal Component Analysis for Protein Folding Dynamics», Journal of Molecular Biology, vol. 385, n. 1, pagg. 312-329, gen. 2009, doi: 10.1016/j.jmb.2008.10.018. 
[55] C. C. David e D. J. Jacobs, «Principal Component Analysis: A Method for Determining the Essential Dynamics of Proteins", Methods Mol Biol, vol. 1084, pagg. 193-226, 2014, doi: 10.1007/978-1-62703-658-0_11.

[56] S. Ebbinghaus et al., "Functional Importance of Short-Range Binding and Long-Range Solvent Interactions in Helical Antifreeze Peptides», Biophysical Journal, vol. 103, n. 2, pagg. L20-L22, lug. 2012, doi: 10.1016/j.bpj.2012.06.013.

[57] K. Meister et al., "Long-range protein-water dynamics in hyperactive insect antifreeze proteins», PNAS, vol. 110, n. 5, pagg. 1617-1622, gen. 2013, doi: 10.1073/pnas.1214911110.

[58] J. Lee, S. Y. Lee, D.-K. Lim, D. J. Ahn, e S. Lee, «Antifreezing Gold Colloids», J. Am. Chem. Soc., vol. 141, n. 47, pagg. 18682-18693, nov. 2019, doi: 10.1021/jacs.9b05526. 